\title{
Autologous matrix-induced chondrogenesis aided reconstruction of a large focal osteochondral lesion of the talus
}

\author{
Martin Wiewiorski • André Leumann · \\ Olaf Buettner • Geert Pagenstert • \\ Monika Horisberger • Victor Valderrabano
}

Received: 17 September 2009/Published online: 21 January 2010

(C) Springer-Verlag 2010

\begin{abstract}
The aim of this case report is to describe a novel technique for treatment of large osteochondral lesions of the talus using autologous matrix-induced chondrogenesis with a collagen I/III membrane.
\end{abstract}

Keywords Osteochondral lesion · Ankle joint · AMIC

\section{Introduction}

Large osteochondral defects of the talus remain a challenging therapeutic task to orthopaedic surgeons. Several surgical techniques are available for treatment, e.g. autologous chondrocyte implantation (ACI), osteochondral autograft transfer system, total talar replacement, matrixinduced autologous chondrocyte implantation (MACI). Good early results are reported; however, literature is limited to case series and case reports and long-term outcome is unknown [1-3].

\footnotetext{
M. Wiewiorski - A. Leumann - O. Buettner - M. Horisberger . V. Valderrabano $(\square)$

Orthopaedic Department, University Hospital Basel,

Spitalstrasse 21, 4031 Basel, Switzerland

e-mail: vvalderrabano@uhbs.ch

M. Wiewiorski

e-mail: mwiewiorski@uhbs.ch
}

\author{
A. Leumann \\ Human Performance Laboratory, Faculty of Kinesiology, \\ University of Calgary, Calgary, Canada \\ G. Pagenstert \\ Orthopaedic Department, University Hospital Bonn, \\ Bonn, Germany
}

This case describes a novel technique for treatment of large osteochondral lesions (OCLs) of the talus utilizing the autologous matrix-induced chondrogenesis (AMIC) technique in combination with a collagen I/III membrane (Chondro-Gide $^{\circledR}$, Geistlich Biomaterials, Wolhusen, Switzerland). The porcine-origin collagen matrix of this membrane stimulates mesenchymal stem cells from the bone marrow of cancellous bone to differentiate into their chondrocytic phenotype, which will produce collagen II and glycosaminoglycans [4].

\section{Patient and methods}

Preoperative management

A 21-year-old man in our outpatient clinic complained about recurrent ankle swelling and load-dependent pain of his right ankle for 1 year. History revealed relapsing ankle sprains. Sport activity was reduced to swimming and cycling. Clinical examination showed discreet swelling of the right ankle and pressure pain at the anteromedial ankle for. Compared to the contralateral side, range of motion (ROM) is slightly reduced with dorsalextension of $15^{\circ}$ and plantarflexion of $35^{\circ}$. The painful ankle presents a maximal anteroposterior and inversion instability $(+++)$ [5], while the deltoid shows stable. Pain measured by the visual analogue scale (VAS: 0 points no pain, 10 point maximal pain; during normal walking) was 5 [6]. The American Orthopaedic Foot and Ankle Society (AOFAS) AnkleHindfoot Scale (score composed of pain, function, and alignment; minimum score 0 points, maximum score 100 points) was poor with 68 points [7].

Initial conventional radiography $(\mathrm{Rx})$ revealed a completely detached but undisplaced OCL at the medial talar 
edge (Berndt/Harty Score 3, Fig. 1a [8, 9]). Magnetic resonance imaging (MRI) presents a partially disrupted cartilage layer of the lesion (Hepple Score 2b, Fig. 1b [10]). Single photon emission computed tomographycomputed tomography (SPECT-CT) reveals the impressive actual size of the posteromedial bony defect with increased remodelling of the lesion itself and surrounding osseous tissue (Ferkel Score 3, Fig. 1c [11]). Integrated hybrid systems like the SPECT-CT are a new approach allowing acquisition of functional SPECT- and anatomical CTimages in a single diagnostic procedure [12]. Due to the large size of the defect $\left(>3 \mathrm{~cm}^{2}\right)$, the young age of the patient and high functional demands, a surgical treatment was chosen in agreement with the patient.

\section{Surgical course}

The patient was positioned supine for anterior approach to the ankle. An initial arthroscopy confirmed the radiologic findings of a large posteromedial lesion. A anteromedial incision was used and the medial malleolus exposed. To gain access to the posteromedial talar edge, a medial malleollar osteotomy was performed. This revealed an large unstable chondral defect $\left(3 \mathrm{~cm}^{2}\right)$ (Fig. 2a). The osteochondral fragment was removed followed by debridement and anterograde drilling of the underlying sclerotic bone (Fig. 2b). Cancellous bone was harvested from the ipsilateral iliac crest and impacted into the defect (Fig. 2c). A template made of aluminium foil was used to make an imprint on the defect. The Chondro-Gide ${ }^{\circledR}$ membrane was cut to the required size according to the template. The membrane was moistened and placed on the defect with the porous layer facing the bone. Fibrin glue was applied at the boundary between the membrane and intact cartilage to close the interface (Fig. 2d). The ankle was then moved several times throughout the whole ROM to control stability. The lateral ankle ligaments where repaired directly anatomically as described by Karlsson et al. [13, 14].

\section{Postoperative care}

Postoperative care consisted of immobilization using a functional orthosis (Vacoped ${ }^{\circledR}$, OPED, Switzerland) with maximum ROM of $30^{\circ}$ and partial weight bearing for 6 weeks $(\max 20 \mathrm{~kg}$ ), followed by progression to full weight bearing under intense physical therapy focusing on strengthening the ankle joint stabilizing lower leg muscles and ROM.

\section{Results}

The patient was available for follow up at 6 weeks, 6 and 12 months, postoperatively. After 6 weeks, the patient had a VAS of 0 , while at 12 weeks he complained about a VAS of 4. This is a regularly encountered course of pain due to begin of full weight bearing and physical therapy after immobilization. At 1 year, the patient was free of pain (VAS 0) during sport activities. He returned to jogging twice a week. No feeling of instability and a new ankle sprains occurred. Clinical examination revealed marginally elevated anteroposterior and inversion instability $(+)$ when compared with the contralateral ankle. The preoperative deficit in ROM remained unchanged. AOFAS hindfoot score increased to the maximum of 100 points. Rx at 1 year showed full osseous consolidation and a nearly anatomic shape of the medial talar edge (Fig. 3).

\section{Discussion}

Large osteochondral defects of the talus remain a difficult therapeutic task to orthopaedic surgeons. This case reports
Fig. 1 Diagnostic imaging. Initial radiography shows a completely fractured but undisplaced OCL at the medial talar edge (a). MRI presents a partially disrupted cartilage layer over the lesion (b). SPECT-CT reveals a large posteromedial bony defect with highly increased uptake on axial $(\#)$ and sagittal $(+)$ reconstruction (c)

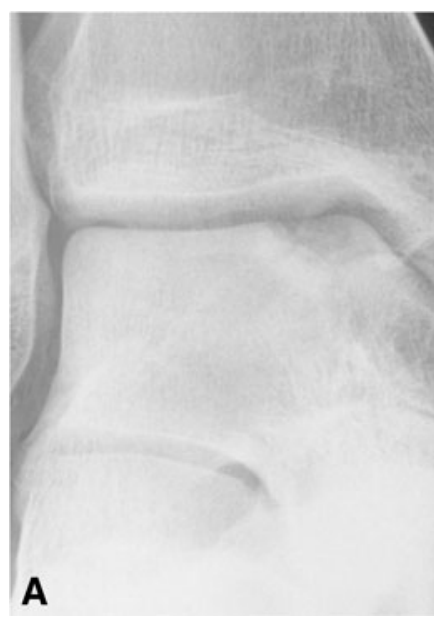

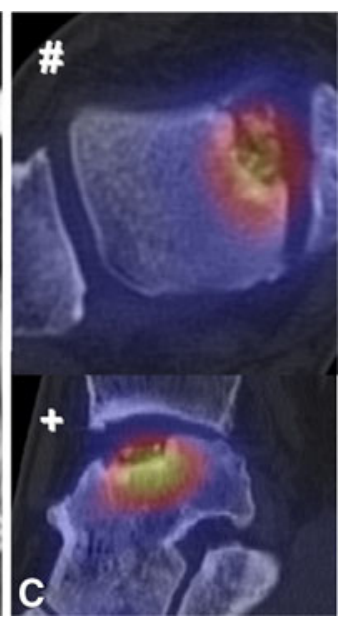


Fig. 2 Surgical procedure. Malleollar osteotomy (\#) reveals an impressive chondral defect at the medial talar edge (a). The osteochondral fragment was removed followed by debridement and anterograde drilling (b). Spongiosa harvested from the iliac crest was impacted into the defect (c). The cut-to-shape collagen membrane is placed on the defect and being glued (d)
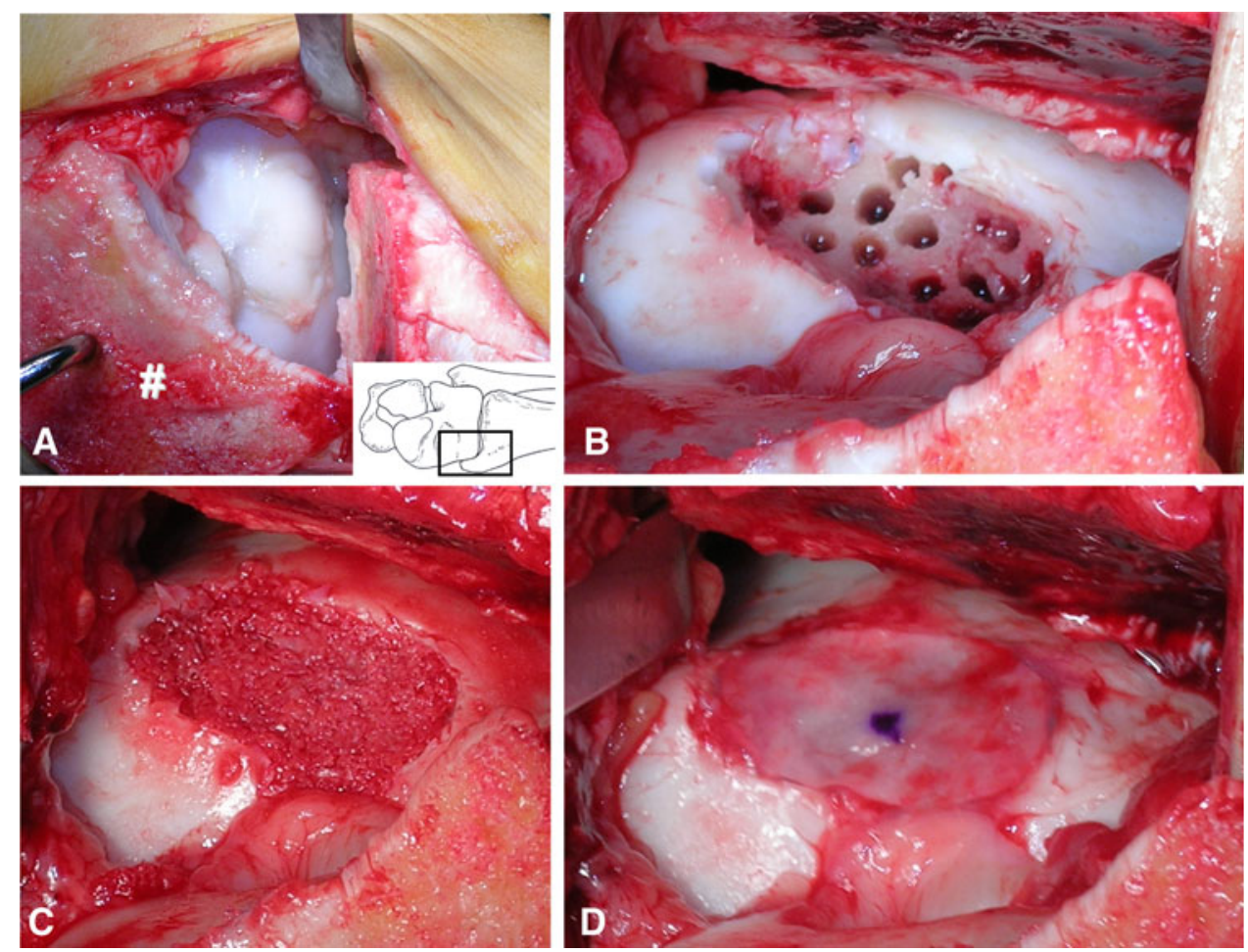

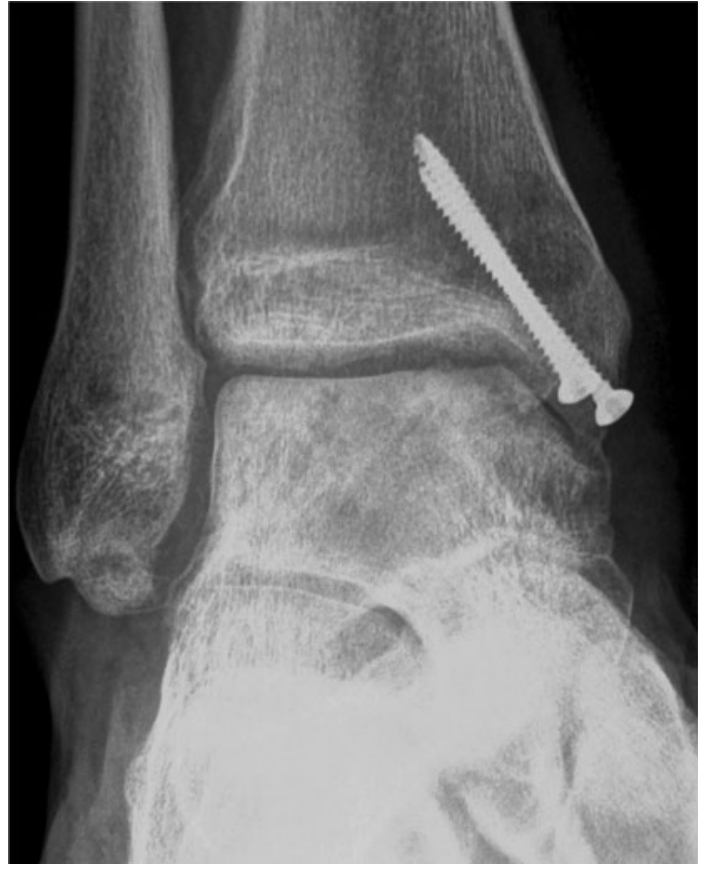

Fig. 3 Radiologic follow-up at 1 year. Conventional radiographs showed full osseous consolidation and a nearly anatomic shape of the medial talar edge

of a new modified marrow stimulation technique with the application of a collagen Type III/I membrane for treatment of severe OCLs of the talus. In this case report, excellent result at 1-year follow-up was seen.
Talar OCL have a huge impact on the patients daily activities as young patients demand to return to sports or hard work. Because of the high demands and chronic progression, patients often undergo surgical treatment.

OCL of the talus are focal and small in size, but include the subchondral bone plate and subchondral bone to a larger extent than similar lesions in the knee joint. In large talar, OCL involvement of the subchondral bone can be found as sclerotic changes, cyst formation, or avascular necrosis [15]. The integrity of the subchondral bone seems to play an important role in the pathogenesis of ankle OCL. In our opinion, in severe and recurrent cases, an optimal treatment includes addressing the subchondral bone, cartilage and mechanical stability of the joint. According to Giannini et al. [1] OCLs of more than $1.5 \mathrm{~cm}^{2}$ can be successfully treated by the MACI. Good clinical outcome has been demonstrated by several authors [16-18].

To the knowledge of the authors, no clinical results for treating talar OCL with the AMIC technique exist. For the AMIC-aided treatment of chondral lesions of the knee joint [19], one author showed good clinical outcome results after treating full-thickness lesions utilizing the above-mentioned collagen membrane combined with ACI at 3-year follow-up [20].

The authors see the following criteria for AMIC-aided debridement and spongiosa-plasty, analogue to surgical criteria for ACI by Giannini et al. [1]: 
- Young patients less than 50 years of age

- Large osteochondral defect of more than $2 \mathrm{~cm}^{2}$ and 0.5 $\mathrm{cm}$ depth

- Failure of previous surgery

- No arthritis

- No axial malalignment (must be corrected, if present)

The AMIC procedure offers following advantages compared to ACI and MACI:

- One step surgical procedure: no culturing after chondrocyte harvesting.

- Less donor site morbidity: no destruction of vital cartilage

- Readily available

- Economically efficient

\section{Conclusion}

Autologous matrix-induced chondrogenesis aided reconstruction of large osteochondral talar lesions shows to be a new promising treatment method. Future research needs to compare this new technique to conventional treatment methods in terms of radiological, clinical and histological outcome.

\section{References}

1. Giannini S, Vannini F (2004) Operative treatment of osteochondral lesions of the talar dome: current concepts review. Foot Ankle Int 25(3):168-175

2. Verhagen RA et al (2003) Systematic review of treatment strategies for osteochondral defects of the talar dome. Foot Ankle Clin 8(2):233-242, viii-ix

3. Giannini S et al (2005) Surgical treatment of osteochondral lesions of the talus in young active patients. J Bone Joint Surg Am 87(Suppl 2):28-41
4. Fuss M et al (2000) Characteristics of human chondrocytes, osteoblasts and fibroblasts seeded onto a type I/III collagen sponge under different culture conditions. A light, scanning and transmission electron microscopy study. Ann Anat 182(4):303310

5. Tohyama $\mathrm{H}$ et al (2003) Anterior drawer test for acute anterior talofibular ligament injuries of the ankle. How much load should be applied during the test? Am J Sports Med 31(2):226-232

6. Price DD et al (1983) The validation of visual analogue scales as ratio scale measures for chronic and experimental pain. Pain $17(1): 45-56$

7. Kitaoka HB et al (1994) Clinical rating systems for the anklehindfoot, midfoot, hallux, and lesser toes. Foot Ankle Int 15(7):349-353

8. Berndt AL, Harty M (1959) Transchondral fractures (osteochondritis dissecans) of the talus. J Bone Joint Surg Am 41A:988-1020

9. Loomer R et al (1993) Osteochondral lesions of the talus. Am J Sports Med 21(1):13-19

10. Hepple S, Winson IG, Glew D (1999) Osteochondral lesions of the talus: a revised classification. Foot Ankle Int 20(12):789-793

11. Ferkel R (1993-1994) Arthroscopic treatment of osteochondral lesions of the talus. Orthop Trans 17:1011

12. Schillaci O (2005) Hybrid SPECT/CT: a new era for SPECT imaging? Eur J Nucl Med Mol Imaging 32(5):521-524

13. Karlsson J et al (1988) Reconstruction of the lateral ligaments of the ankle for chronic lateral instability. J Bone Joint Surg Am 70(4):581-588

14. Brostrom L (1966) Sprained ankles. VI. Surgical treatment of "chronic" ligament ruptures. Acta Chir Scand 132(5):551-565

15. Barnes CJ, Ferkel RD (2003) Arthroscopic debridement and drilling of osteochondral lesions of the talus. Foot Ankle Clin 8(2):243-257

16. Ronga M, Grassi FA (2005) Treatment of deep cartilage defects of the ankle with matrix-induced autologous chondrocyte implantation (MACI). Foot Ankle Surg 11:29-33

17. Behrens P et al (2006) Matrix-associated autologous chondrocyte transplantation/implantation (MACT/MACI) -5-year follow-up. Knee 13(3):194-202

18. Horst F, Gilbert BJ, Nunley JA (2004) Avascular necrosis of the talus: current treatment options. Foot Ankle Clin 9(4):757-773

19. Behrens P (2005) Matrixgekoppelte Mikrofrakturierung. Arthroskopie 18:193-197

20. Steinwachs M, Kreuz PC (2007) Autologous chondrocyte implantation in chondral defects of the knee with a type I/III collagen membrane: a prospective study with a 3-year follow-up. Arthroscopy 23(4):381-387 